\title{
Multi-colour optical monitoring of the intra-day variable blazar S5 0716+71*
}

\author{
R. Sagar $^{1,2}$, Gopal-Krishna ${ }^{3}$, V. Mohan ${ }^{1}$, A.K. Pandey ${ }^{1}$, B.C. Bhatt ${ }^{1,2}$, and S.J. Wagner ${ }^{4}$ \\ 1 U.P. State Observatory, Manora Peak, Naini Tal, India \\ e-mail: sagar@upso.ernet.in \\ 2 Indian Institute of Astrophysics, Bangalore 560034, India \\ 3 N.C.R.A.-T.I.F.R., Pune University Campus, Pune-411007, India \\ 4 Landessternwarte Heidelberg-Koenigstuhl, 69117 Heidelberg, Germany
}

Received July 28; accepted August 31, 1998

\begin{abstract}
We report multi-colour optical monitoring of the intra-day variable blazar S5 0716+71 with a dense temporal coverage over a total duration of 4 weeks in February/March 1994. Four filters ( $B V$ Johnson and $R I$ Cousins) were used on a majority of the nights. The light curves of the blazar were derived relative to two comparison stars present on the CCD frames. Intensity variations of $\geq 0.05$ mag from night-to-night were observed in half the cases and a good correlation between the light curves in the different passbands was noticed (as also for the observed intra-night variations). There seems to be no correlation between flux and optical spectral index of the blazar. In all, three prominent events of intra-night variability were detected, each with a rate of $\geq 0.1 \mathrm{mag}$ per hour, lasting for at least 3 hours. Each of these flaring segments of the blazar light curves can be well fitted with an exponential intensity profile whose rate of variation is essentially the same for all the passbands. No clear event of ultra-rapid fluctuations of the blazar on time scale $\leq 1$ hour was detected in these multi-colour data. Present observations have been carried out during a low brightness level of the blazar and they have no large amplitude variable events.
\end{abstract}

Key words: BL Lac objects: general - BL Lac objects: individual: S5 0716+71 - galaxies: photometry

Send offprint requests to: R. Sagar, Naini Tal

* Based on observations obtained at the U.P. State Observatory, Naini Tal, India and V.B. Observatory, Kavalur, India.

\section{Introduction}

Study of rapid intensity variations of active galactic nuclei provides a uniquely powerful tool for investigating the processes occurring in the vicinity of their central engines. This strategy is further sharpened when the variability is monitored simultaneously in different frequency bands. The flat-spectrum radio source S5 $0716+71$, which is an intra-day variable at radio and optical wavelengths as well as a $\gamma$-ray emitter (Wagner et al. 1996) is a favourite target for variability studies. This BL Lac object (Biermann et al. 1981) is located at a redshift $z \geq 0.3$ (Schalinski et al. 1992) and its radio structure shows a double structure reminiscent of the FR II morphology (Antonucci et al. 1986). Remarkably, in a 4-week long monitoring campaign in February 1990, this blazar showed an abrupt transition in its quasi-periodic variability pattern from $\sim 1$-day timescale during the first week, to $\sim 6$-day timescale subsequently, both at optical and centimetre wavelengths (Quirrenbach et al. 1991; Wagner 1991). This strong correlation between the optical and centimetre regimes provided a strong hint that the observed rapid radio variability is not due to propagation effects in the intervening media. Another interesting result is that the radio spectral index of this blazar was found to correlate with intra-night optical variations, such that the radio spectrum flattens near the optical maxima for several cycles (Qian et al. 1991; Wagner et al. 1996). Tendency for the optical spectrum $(B-I)$ to harden during the bright optical phase has been reported in Wagner et al. (1996). These authors further inferred that in the $R$-band light curves a significant signature of "flickering" on time scale as short as $\approx 15 \mathrm{~min}$, i.e., much faster than the observed quasi-periodicity on $\sim 1$ day time scale.

Recently, Ghisellini et al. (1997) have reported results of monitoring of this blazar in the $B, V, R, I$ colours during 
November 1994 to April 1995. They have also provided in these passbands the calibrated magnitudes of 4 comparison stars in the field of this blazar for which they have estimated an interstellar extinction of $A_{V}=0.23$ mag. Their campaign recorded a few events of ultra-rapid (intranight) variations with an amplitude of about $5 \%$ within a few hours, superposed on slower variations. Another finding from their study is that in the "low" optical state the $(B-R)$ spectral index responds to fast optical variations occurring on day-like time scales, the spectrum becoming bluer with increasing brightness in $R$. Interestingly, such a correlation was not found for the slower variations, indicating a different physical mechanism for such variations (Ghisellini et al. 1997).

In order to investigate further this blazar, a onemonth long multi-band monitoring campaign covering radio through $\gamma$-ray bands was carried out by us and our collaborators during February/March 1994. In the optical, only $R$ band was common to all the datasets obtained from the various participating observatories. A detailed presentation based on this campaign is under preparation. In this brief paper, we confine ourselves to presenting the results from our dense multi-colour optical monitoring. These observations covered a total baseline of one month and provided a fairly dense coverage on most of the nights. Such data can allow us to resolve the temporal structure of the intensity variations, thereby providing a closer insight into the radiation processes involved.

\section{Observations and data reduction}

The multi-star CCD photometric monitoring was carried out for 21 nights during February/March 1994 using two 1-metre class telescopes located at Naini Tal and Kavalur in India, at a separation of $\sim 2500 \mathrm{~km}$. Due to the high declination of the blazar the airmass was generally larger than 1.5 , but the object could be monitored typically for about 5 hours in a night. The positional offsets, the calibrated $B V$ Johnson and $R I$ Cousins magnitudes and instrumental colours of the three comparison stars used for differential photometry of the blazar are listed in Table 1 . The photometric measurements for stars 1 and/or 2 have been carried out by Takalo et al. (1994); Ghisellini et al. (1997) and Villata et al. (1998) in the different passbands. Within the errors, the magnitudes derived by them for a star agree fairly well. For stars 1 and 2, the $B V R$ data are taken from Villata et al. (1998) as they are the most accurate measurements; while the $I$ magnitudes are adopted from Ghisellini et al. (1997). For star 3, these values are determined from stars 1 and 2, using the differential instrumental magnitudes measured in our campaign. A log of observations for the entire campaign is given in Table 2 . Further details of the observations are summarized below.
Table 1. Positional offsets in arcmin and the differential $(\Delta)$ instrumental $(b-v)$ and $(v-i)$ colours in mag of the comparison stars are relative to the blazar S5 0716+71. Stars observed by Takalo et al. (1994), Ghisellini et al. (1997) and Villata et al. (1998) have been identified with prefix T-, G- and Vrespectively. $B V R I$ are standard magnitudes

\begin{tabular}{|c|c|c|c|}
\hline & Star 1 & Star 2 & Star 3 \\
\hline $\begin{array}{l}\text { Other ide- } \\
\text { ntifications }\end{array}$ & $\begin{array}{l}\text { G-C, V-5, } \\
\text { T-2 }\end{array}$ & G-B, V-3 & \\
\hline Offset $(\mathrm{E} / \mathrm{W})$ & $0.04 \mathrm{E}$ & $0.10 \mathrm{~W}$ & $0.92 \mathrm{~W}$ \\
\hline Offset (N/S) & $1.15 \mathrm{~S}$ & $2.10 \mathrm{~S}$ & $1.28 \mathrm{~S}$ \\
\hline$\Delta(b-v)$ & 0.13 & 0.13 & 0.19 \\
\hline$\Delta(v-i)$ & -0.20 & -0.24 & -0.18 \\
\hline$B$ & $14.15 \pm 0.01$ & $13.04 \pm 0.01$ & $15.69 \pm 0.03$ \\
\hline$V$ & $13.55 \pm 0.02$ & $12.43 \pm 0.02$ & $16.16 \pm 0.04$ \\
\hline$R$ & $13.18 \pm 0.01$ & $12.06 \pm 0.01$ & $15.75 \pm 0.03$ \\
\hline$I$ & $12.85 \pm 0.05$ & $11.79 \pm 0.05$ & $15.39 \pm 0.06$ \\
\hline
\end{tabular}

Table 2. Log of the CCD observations

\begin{tabular}{cccl}
\hline Date & Observatory & $\begin{array}{c}\text { Duration } \\
\text { (hours of UT) }\end{array}$ & Filter(s) \\
\hline 28 Feb. 94 & UPSO & $15.5-16.3$ & $B, V, R$ \\
1 Mar. 94 & UPSO & $14.5-19.2$ & $B, V, R, I$ \\
2 Mar. 94 & UPSO & $13.5-19.9$ & $B, V, R, I$ \\
3 Mar. 94 & UPSO & $14.4-19.7$ & $B, V, R, I$ \\
4 Mar. 94 & UPSO & $14.6-19.5$ & $B, V, R, I$ \\
5 Mar. 94 & UPSO & $14.6-18.4$ & $B, V, R, I$ \\
6 Mar. 94 & UPSO & $14.6-19.4$ & $B, V, R, I$ \\
7 Mar. 94 & UPSO & $14.8-19.5$ & $B, V, R, I$ \\
8 Mar. 94 & UPSO & $13.9-19.1$ & $B, V, R, I$ \\
15 Mar. 94 & UPSO & $15.4-17.8$ & $B, V, R, I$ \\
16 Mar. 94 & UPSO & $14.4-18.8$ & $B, V, R, I$ \\
16 Mar. 94 & VBO & $14.2-19.2$ & $R, I$ \\
17 Mar. 94 & UPSO & $15.6-18.4$ & $B, V, R, I$ \\
17 Mar. 94 & VBO & $14.3-18.6$ & $R, I$ \\
18 Mar. 94 & UPSO & $15.2-18.2$ & $B, V, R, I$ \\
18 Mar. 94 & VBO & $13.8-18.6$ & $R, I$ \\
19 Mar. 94 & VBO & $13.8-18.6$ & $R, I$ \\
20 Mar. 94 & VBO & $14.2-18.0$ & $R, I$ \\
21 Mar. 94 & UPSO & $15.1-18.1$ & $B, V, R, I$ \\
22 Mar. 94 & UPSO & $13.8-18.2$ & $B, V, R, I$ \\
25 Mar. 94 & UPSO & $14.3-16.4$ & $B, V, R, I$ \\
26 Mar. 94 & UPSO & $14.4-16.1$ & $V, R, I$ \\
27 Mar. 94 & UPSO & $14.4-16.0$ & $V, R, I$ \\
28 Mar. 94 & UPSO & $14.2-15.2$ & $V, R, I$ \\
\hline
\end{tabular}




\subsection{The UPSO observations}

The $B V$ Johnson and $R I$ Cousins photometric observations were carried out using the Photometrics CCD system at the $\mathrm{f} / 13$ Cassegrain focus of the 104-cm Sampurnanand reflector of the Uttar Pradesh State Observatory (UPSO), Naini Tal on 19 nights in February/March 1994 (Table 2). Typical seeing was around $1.5^{\prime \prime}$. Details of the CCD system are reported in Mohan et al. (1991). Typical exposure times were $20-60 \mathrm{~s}$ in $R$ and $I, 1-2 \mathrm{~min}$ in $V$ and $3-5 \mathrm{~min}$ in $B$. The observed comparisons are stars 1 and 2 (Table 1).

\subsection{The $V B O$ observations}

CCD photometric observations in $R$ and $I$ Cousins passbands were made using the Thomson-CSF TH7882 CCD chip mounted at the $\mathrm{f} / 13$ Cassegrain focus of the 102-cm telescope of the Vainu Bappu Observatory (VBO) at Kavalur. Typical seeing was around $2^{\prime \prime}$. Details of the instrumental set up can be found in Sagar \& Pati (1989). Typical exposure times were $5-10 \mathrm{~min}$ in both $R$ and $I$ passbands (owing to large zenith distance of the object and frequent cloudy conditions). The observed comparisons are stars 1 and 3 (Table 1 ).

\subsection{The differential light curves (DLCs)}

At both sites, bias and dark frames were taken intermittently throughout the observations. The pixel size of $23 \times 23(\mu \mathrm{m})^{2}$ of both CCD systems corresponds to 0.36 $(\operatorname{arcsec})^{2}$ on the sky. The chip size of $384 \times 576$ pixels covers an area of $2.3 \times 3.4(\operatorname{arcmin})^{2}$. In order to improve the signal/noise ratio the data were acquired in the binning mode of $2 \times 2$ (pixels) $)^{2}$. In order to detect small intensity fluctuations, we performed differential photometry, generally ensuring that the locations of the blazar and the comparison stars did not change by more than a few pixels from exposure to exposure during a night. This largely obviated the need to flat-field the frames (which introduces additional noise). The background was subtracted using the measured counts within the annuli (between radii of 25 and 35 pixels) around the circular aperture of 5 pixel radius, centred at the desired object.

The data were reduced at the VBO VAX 11/780 system using DAOPHOT software package, as described in Gopal-Krishna et al. $(1993 ; 1995)$. In order to maximise the signal/noise ratio, we performed the aperture-growth analysis described by Howell (1989). Accordingly, a circular aperture of 10 pixels $\left(=7^{\prime \prime}\right)$ diameter was adopted for the photometry of the blazar as well as the comparison stars. The DAOPHOT algorithm also provided estimates of the statistical errors of the computed relative magnitudes, which were usually $<1 \%$ for all the passbands. Using the computed instrumental magnitudes, differential light curves (DLCs) were generated. These represent the relative instrumental magnitudes of the blazar relative to the two comparison stars, and the same for the pair of comparison stars (Figs. 1 and 2). Note that the comparisons used are stars 1 and 2 for the UPSO DLCs and stars 1 and 3 for the VBO DLCs. It is seen that the average instrumental colours of the blazar are not much different from those of the three comparison stars (Table 1).

\section{Results}

On 13 out of the total 21 nights, the DLCs span a minimum duration of 4 hours and have a fairly dense coverage (about 4 data points per hour in each passband). Of the 5 nights observed from VBO, three (March 16, 17 and 18) overlap with the monitoring carried out from UPSO. Fortunately, the two cloudy nights at UPSO (March 17 and 18) happened to fall within this overlap and this deficit can thus be partly offset with the available VBO data. The March 16 data from both observatories have high signal/noise ratio and are found to be in good agreement (Figs. 1 and 2). Note that all 4 passbands have been used on 15 of the nights, whereas only $R$ and $I$ data are available for the two nights covered from VBO alone. Also, on 4 nights at UPSO only 3 filters $(V, R, I)$ were used.

In order to examine inter-night and long-term optical variability trends more clearly, we have generated a compressed version of the DLCs, integrating just the data acquired during the first and the last hour of observations on each night. This gave two data points per night (excepting a couple of cases when the entire duration of observation was less than two hours and therefore only one data point could be obtained). The above integration typically involved 3-4 data points in $B$ and $V$, and 5-6 data points in $R$ and $I$ passbands. The compressed DLCs are shown in Fig. 3.

\section{Discussions}

\subsection{Long-term optical variability}

Present light curves (Fig. 3) in combination with the optical light curves given by Wagner et al. (1996) and by Ghisellini et al. (1997) are used to decipher behaviour of the long-term optical variability of the blazar. The 8 months (in 1990/1991 winter) long relative light curve given by Wagner et al. (1996) shows a variation of about $2.5 \mathrm{mag}$ in $R$ passband. The source remained in "low" state for about 50 days starting from JD $=2448150$. Then, after a slight brightening, it declined to a minimum intensity level around JD $=2448270$. After that it brightened $(\sim 2.5 \mathrm{mag}$ in $R$ ) rapidly to reach a maximum intensity level around JD $=2448300$. On the other hand, over the 5 months (November 1994 to April 1995) observational period, the light curve in $R$ passband given 


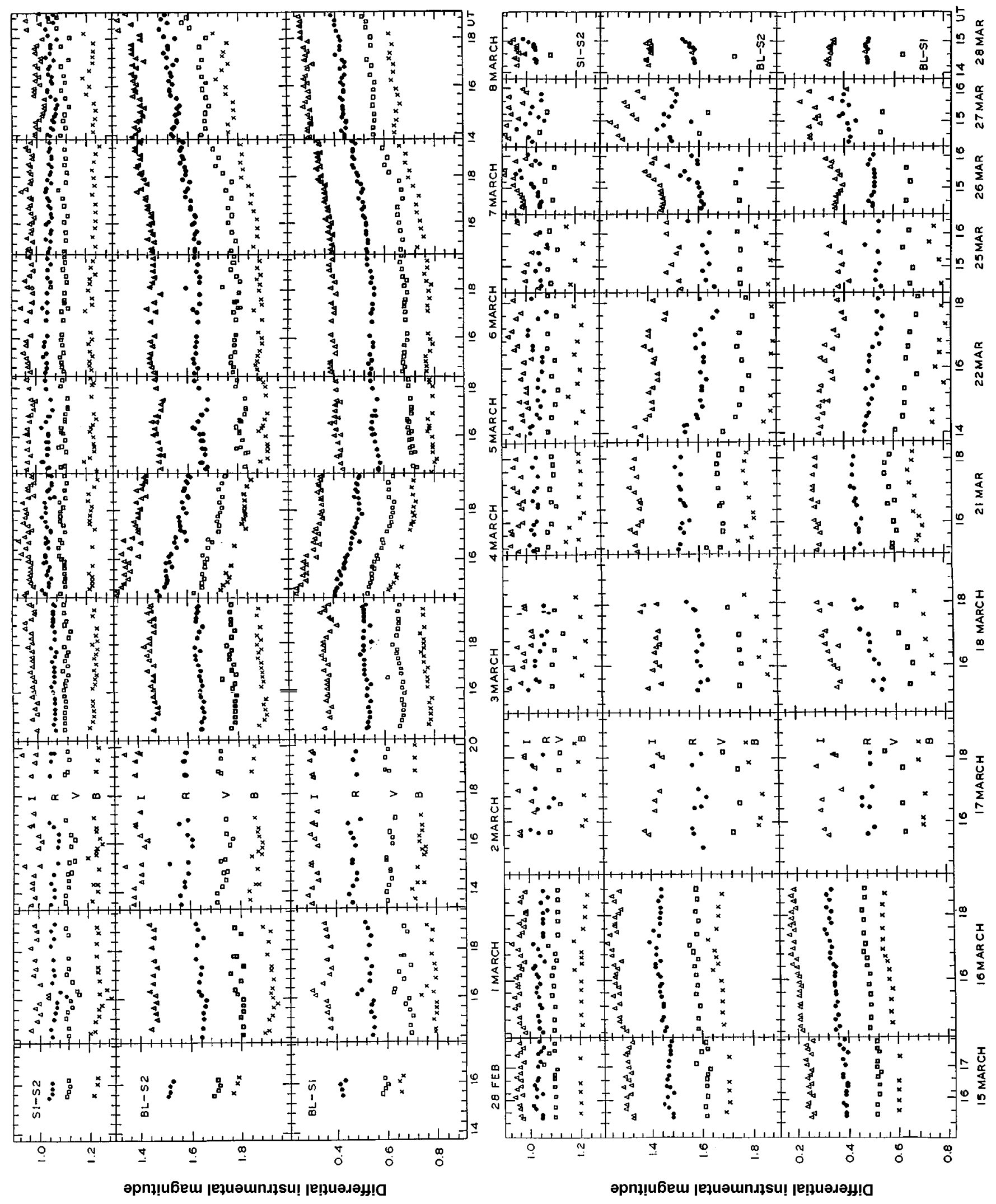

Fig. 1. DLCs in the $B, V, R, I$ passbands deduced from the UPSO observations on 19 nights (excepting for February 28 on which $I$-band data could not be obtained, whereas for the last 3 nights $B$-band observations could not be made). The symbols crosses, squares, filled circles and triangles refer to $B, V, R$ and $I$ passbands respectively. In order to plot the DLCs with clarity in the $B, V, R, I$ sequence from bottom to top, we have applied fixed offsets of 0.25 mag in $B, 0.0$ mag in $V$ and -0.1 mag in $R$ and $I$ for the DLCs involving the blazar and $0.1 \mathrm{mag}$ in $B, 0.0 \mathrm{mag}$ in $V$ and $-0.05 \mathrm{mag}$ in $R$ and $-0.1 \mathrm{mag}$ in $I$ for the DLCs involving the two comparison stars 1 and 2 


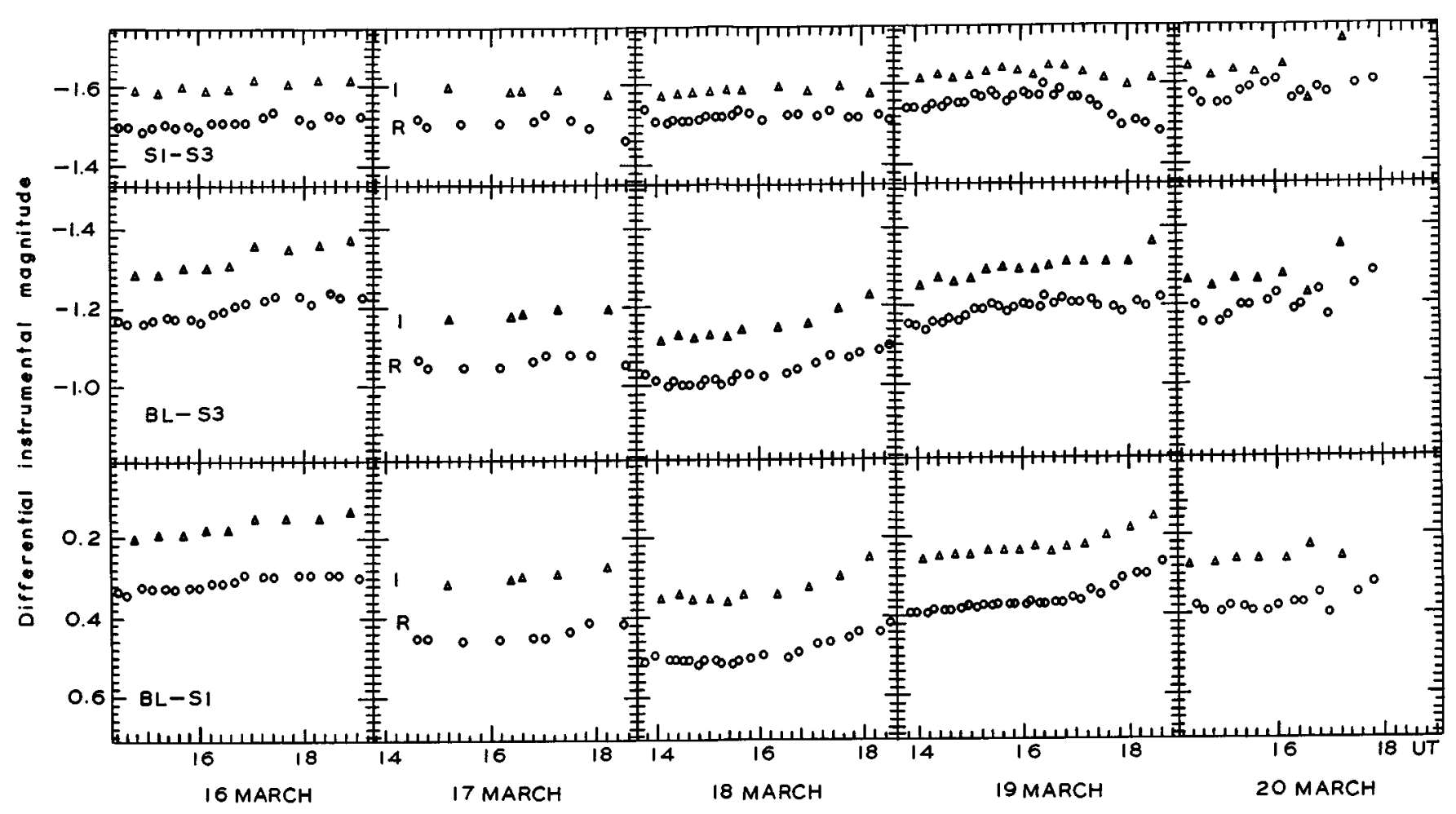

Fig. 2. DLCs in the $R$ and $I$ passbands obtained at VBO for 5 consecutive nights. The temporal overlap with the UPSO data on the first 3 nights (Fig. 1) shows good consistency between the two datasets. Note that the comparison star 1 is common to the two datasets. For clarity in plot, the offsets applied are $-0.1 \mathrm{mag}$ in both $R$ and $I$ for the DLCs involving the blazar, and $-0.1 \mathrm{mag}$ in $I$ and $0.0 \mathrm{mag}$ in $R$ for the DLCs involving the two comparison stars 1 and 3

by Ghisellini et al. (1997) shows that the source brightened after JD $=2449720$, remained bright for $\sim 50$ days, and then faded rapidly to reach a minimum intensity level around JD $=2449800$. Thus in the long-term optical variability of the blazar, events with rapid brightening as well as fading (only one in both cases) have been observed in the above mentioned two $R$ passband light curves. It has to be noted that the durations of both events are almost the same about 30 days.

The average calibrated $B, V, R$ and $I$ magnitudes of the blazar during present observations are $\sim 14.7,14.2$, 13.8 and 13.3 respectively. A comparison of these numbers and the present light curve (Fig. 4) with optical light curves given by Ghisellini et al. (1997) indicates that during the entire period ( $\sim 30$ days) of our campaign, the blazar was in a relatively low state without any rapid large amplitude ( $\geq 1 \mathrm{mag}$ ) brightening or fading as the exhibited maximum variations are only $\sim 0.2 \mathrm{mag}$ in all four passbands.

\subsection{Inter-night variability}

As seen from Fig. 3, the blazar remained moderately active during the 4 -weeks of monitoring, the observed variations often being much larger than the rms scatter of the relative magnitudes of the pair of comparison stars, which is
Table 3. Clearly defined outbursts of the blazar S5 0716+71

\begin{tabular}{ccc}
\hline $\begin{array}{c}\text { Date } \\
\text { March } 94\end{array}$ & $\begin{array}{c}\text { Amplitude } \\
\text { (mag) }\end{array}$ & $\begin{array}{c}\text { Duration } \\
\text { (days) }\end{array}$ \\
\hline 2 & 0.08 & 2 \\
4 & 0.14 & 2 \\
7 & 0.20 & 4 \\
18 & 0.24 & 4 \\
26 & 0.12 & 2 \\
\hline
\end{tabular}

found to be $\sim 0.014$ mag for all the four passbands. Table 3 lists the date, amplitude and duration of those outbursts whose profiles are clearly defined. There seems to be a weak correlation between amplitude and duration of the flares in the sense larger the amplitude longer its duration. For shorter duration flares, the rising and decaying times appear approximately equal and profiles are symmetric with no indications of a "plateau" longer than one day. Similar behaviour has also been noticed by Ghisellini et al. (1997). For the largest outburst, the profile is asymmetric with rising time about one day and decaying time about 3 days.

From the DLCs of all 4 passbands, it appears that the blazar outbursts are superposed on a constant base level emission. This behaviour differs from those observed during the extended optical monitoring campaigns of Wagner 


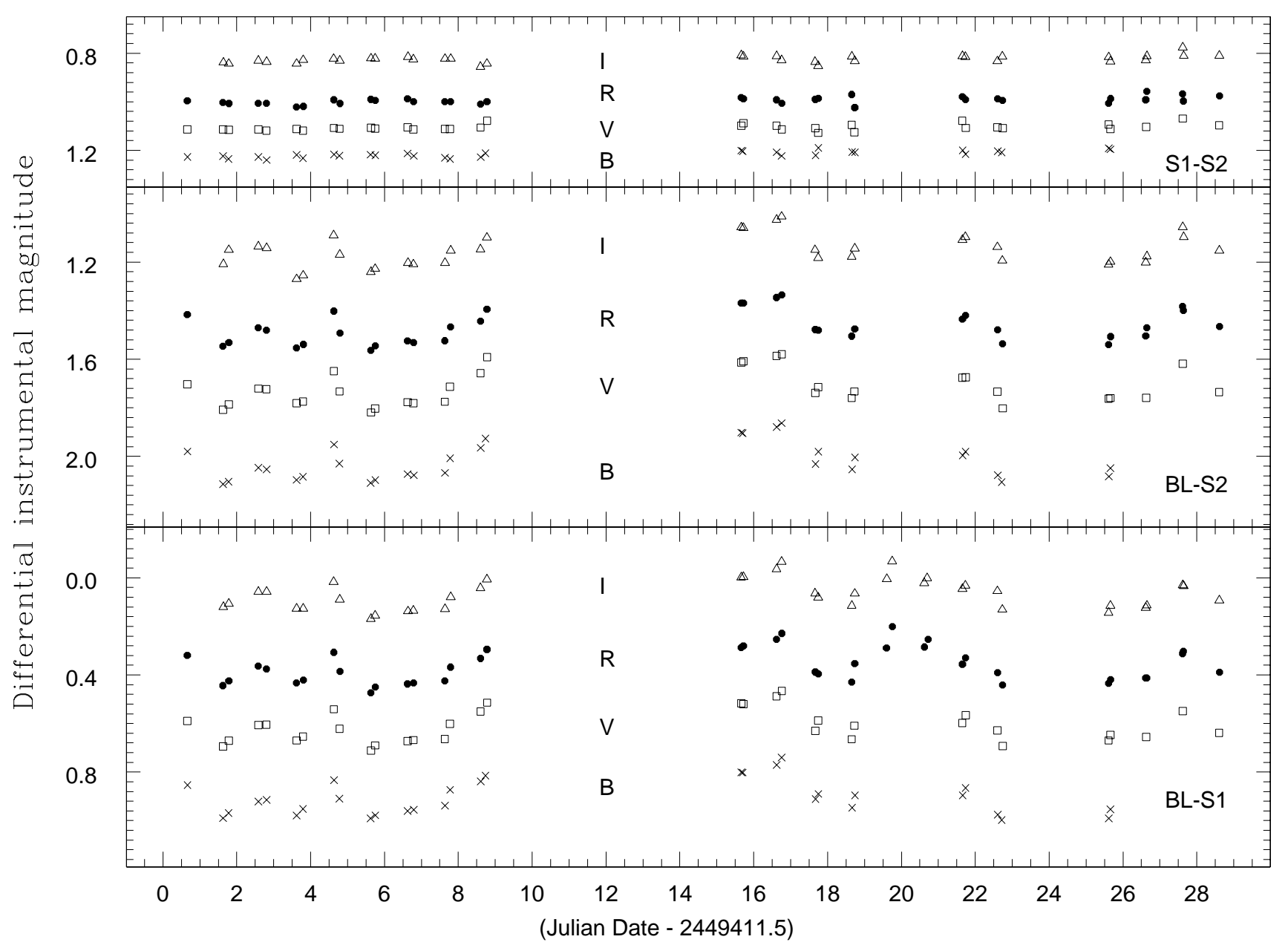

Fig. 3. Compressed DLCs generated from the profiles shown in Figs. 1 and 2. These DLCs represent only the data acquired during the first and the last hour of the individual nights. In order to enhance clarity, we have applied fixed offsets of 0.45 , $0.0,-0.2$ and -0.35 magnitudes, respectively, to the $B, V, R$ and $I$-band DLCs of the blazar. The corresponding offsets for the DLCs of the comparison stars are $0.1,0.0,-0.1$ and -0.25 magnitudes, respectively. Note that the gap in the $R$ and $I$ data of UPSO on March 19 and 20 has been filled using the VBO observations, since one of the comparisons star 1 was common to the two datasets

et al. (1996) and Ghisellini et al. (1997) when the base levels were found to either drop from a "high" to a "low" state or vice-versa by almost a factor of 2 or so within about a day (see also Quirrenbach et al. 1991). The quasiperiodicity of the optical light curve with a characteristic time scale of 1 day, which was then found to persist for a week is not clearly evident during our observations, only some indication of it is present during the first week (Fig. 3). A rigorous comparison of the present data with the earlier dataset would be difficult, because the location of this blazar restricted the monitoring from India during the month of March to just 4-6 hours per night, at most, thereby leaving large gaps in the light curves. It is clear, nonetheless, that in all four passbands the blazar exhibited variations of up to $\sim 0.2$ mag between two consecutive nights, as measured relative to both comparison stars. Furthermore, the light curves in the four passbands are tightly correlated, indicating that the spectrum of the flaring component is not much different from that of the "base" emission. Recall that a close similarity of the light curves at $R, V$ and $B$ during the quasi-periodic variations was also noticed during the 1990 campaign (Quirrenbach et al. 1991; Wagner et al. 1996). However, the campaign by Ghisellini et al. (1997) indicates that in the "low" optical state of the blazar, the $(B-R)$ spectral index responds to fast optical variations occurring on day-like time scales, the spectrum becoming bluer with increasing brightness in $R$. 


\subsection{Flux-optical spectral index correlation}

In order to study the correlation between flux and optical spectral index $\left(\alpha_{B I}\right)$ defined by the $B V R I$ values of present observations, we plot the standard $R$ magnitude and $\alpha_{B I}$ against time in Fig. 4. We determined the $R$ values from the compressed DLCs presented in Fig. 3 and the calibrated $R$ magnitude of star 1 given in Table 1 . The spectral index $\alpha_{B I}$ has been calculated by a least-square linear fit procedure, after dereddening the observed calibrated flux of the blazar in $B V R I$ passbands. For this, following Ghisellini et al. (1997), the reddening corrections were taken as $A_{B}=0.30, A_{V}=0.23, A_{R}=0.19$ and $A_{I}=0.14$ magnitudes. The regression coefficients of the linear fit are always $\sim 1$. The values of $\alpha_{B I}$ obtained in this way vary from 0.81 to 1.01 with a mean value of $0.84 \pm 0.043$, and do not have any significant correlation with the flux (see Fig. 4). Ghisellini et al. (1997) have also obtained a similar result. However, the values of their spectral index, $\alpha_{B R}$ have some what larger range (from 0.81 to 1.15 ) and higher mean value, 0.94 .

\subsection{Intra-night variability}

An important issue in the variability studies concerns the shape(s) of the profiles of the flares. For some of the densely monitored blazars, the large individual optical flares seen on the time-scale of $\approx 1$ day are characterized by exponential growth and decay profiles, as, e.g., in the cases of the intra-day variable blazars PKS 2155-304 (Urry et al. 1993) and S5 0954+658 (Wagner et al. 1993). In contrast, the individual flares on day-like time scale recorded in 1990 and 1994-95 optical monitoring of the present blazar S5 $0716+71$ are well described by linear profiles (cf. Wagner et al. 1996; Ghisellini et al. 1997). Since any dichotomy of this nature can signify fundamentally different emission processes, we have made an attempt to determine the shapes of the individual optical flares of S5 $0716+71$ recorded during our campaign.

As seen from Figs. 1 and 2, the blazar showed on 3 nights prominent optical flares with rates $\gtrsim 0.01$ mag per hour, sustained over a minimum of two hours (see also, Fig. 5). Note that the positive bump peaking around $19^{\mathrm{h}}$ UT on the DLCs of March 4 is clearly related to the variation of the comparison star 1 (Fig. 1) and hence we have ignored the affected portion of the blazar DLC from subsequent analysis. Since all three flares indicate a linear temporal variation on the magnitude scale, we have fitted least-square regression lines to these flaring segments of the DLCs. These best-fit lines are shown in Fig. 5 and their slopes are given in Table 4, together with the regression coefficients. It is interesting that for each flare the slopes are essentially the same for the different passbands. The difference in the slopes is always $\leq 1 \sigma$. It is therefore not statistically significant. Moreover, the regression coefficients
Table 4. Slopes and regression coefficients $(\gamma)$ of the leastsquare linear fits to the intra-night magnitude variations

\begin{tabular}{cccccc}
\hline $\begin{array}{c}\text { Date } \\
\text { March } 94\end{array}$ & Filter & DLC1 & \multicolumn{2}{c}{ DLC2 } \\
\hline 4 & & slope $\pm \sigma$ & $\gamma$ & slope $\pm \sigma$ & $\gamma$ \\
\hline & $V$ & $0.030 \pm 0.002$ & 0.99 & $0.024 \pm 0.002$ & 0.95 \\
& $R$ & $0.030 \pm 0.002$ & 0.99 & $0.024 \pm 0.002$ & 0.95 \\
& $I$ & $0.028 \pm 0.003$ & 0.96 & $0.022 \pm 0.002$ & 0.93 \\
7 & $B$ & $-0.020 \pm 0.002$ & 0.92 & $0.020 \pm 0.002$ & 0.89 \\
& $V$ & $-0.017 \pm 0.004$ & 0.80 & $-0.017 \pm 0.003$ & 0.84 \\
& $R$ & $-0.015 \pm 0.001$ & 0.96 & $-0.015 \pm 0.001$ & 0.94 \\
& $I$ & $-0.014 \pm 0.001$ & 0.96 & $-0.014 \pm 0.001$ & 0.89 \\
& & & & & \\
18 & $B$ & $-0.027 \pm 0.004$ & 0.97 & $-0.030 \pm 0.010$ & 0.88 \\
& $V$ & $-0.027 \pm 0.008$ & 0.88 & $-0.018 \pm 0.008$ & 0.77 \\
& $R$ & $-0.035 \pm 0.004$ & 0.94 & $-0.014 \pm 0.008$ & 0.88 \\
& $I$ & $-0.028 \pm 0.006$ & 0.78 & $-0.010 \pm 0.008$ & 0.37 \\
\hline
\end{tabular}

for the linear fits are $\geq 90 \%$ in all cases, excepting the $I$-band profile on March 18 which is noisy, as evident from the DLC of the comparison stars (Fig. 1). Thus, at least over the few hours spanned by these observations, all the three prominent events of intra-night variability can be described by linear trends on magnitude scale (which, therefore, corresponds to an exponential intensity variation). Similar linear intra-night magnitude variations in $B$ and $R$ bands have been noticed by Ghisellini et al. (1997) on two occasions. Unfortunately, none of the flaring segments of the present DLCs encompass the intensity turnover point (which is not unexpected considering the modest durations of these DLCs). Therefore, the question of linear vis-a-vis exponential variation cannot yet be settled conclusively, though an exponential intensity variation seems to be consistent with all the prominent intra-night flares recorded in the present as well as Ghisellini et al. (1997) optical observations of S5 $0716+71$. It would be of great interest to enquire if the shapes (linear/exponential) of the outbursts of a given blazar depend on the time-scale of the outburst and whether they vary from one occasion to another.

\subsection{Search for ultra-rapid fluctuations}

Since on many nights in the present campaign, a fairly dense temporal coverage was achieved simultaneously in the different passbands, it is possible to look for any ultrarapid fluctuations on time scales shorter than $\sim 1$ hour. In particular, such events can provide important clues to relativistic beaming of the AGN's optical radiation (e.g., Guilbert et al. 1983). Indeed, based on the 1990 campaign substantial flickering of this blazar on time scales as short as $\sim 15$ min was inferred from the structure function analysis of the optical light curve (Wagner et al. 1996). A close look at Fig. 1 reveals many events of ultra-rapid fluctuations, often occurring in more than one passband simultaneously. Table 5 provides a log of such individual events which were detected during almost every observing 


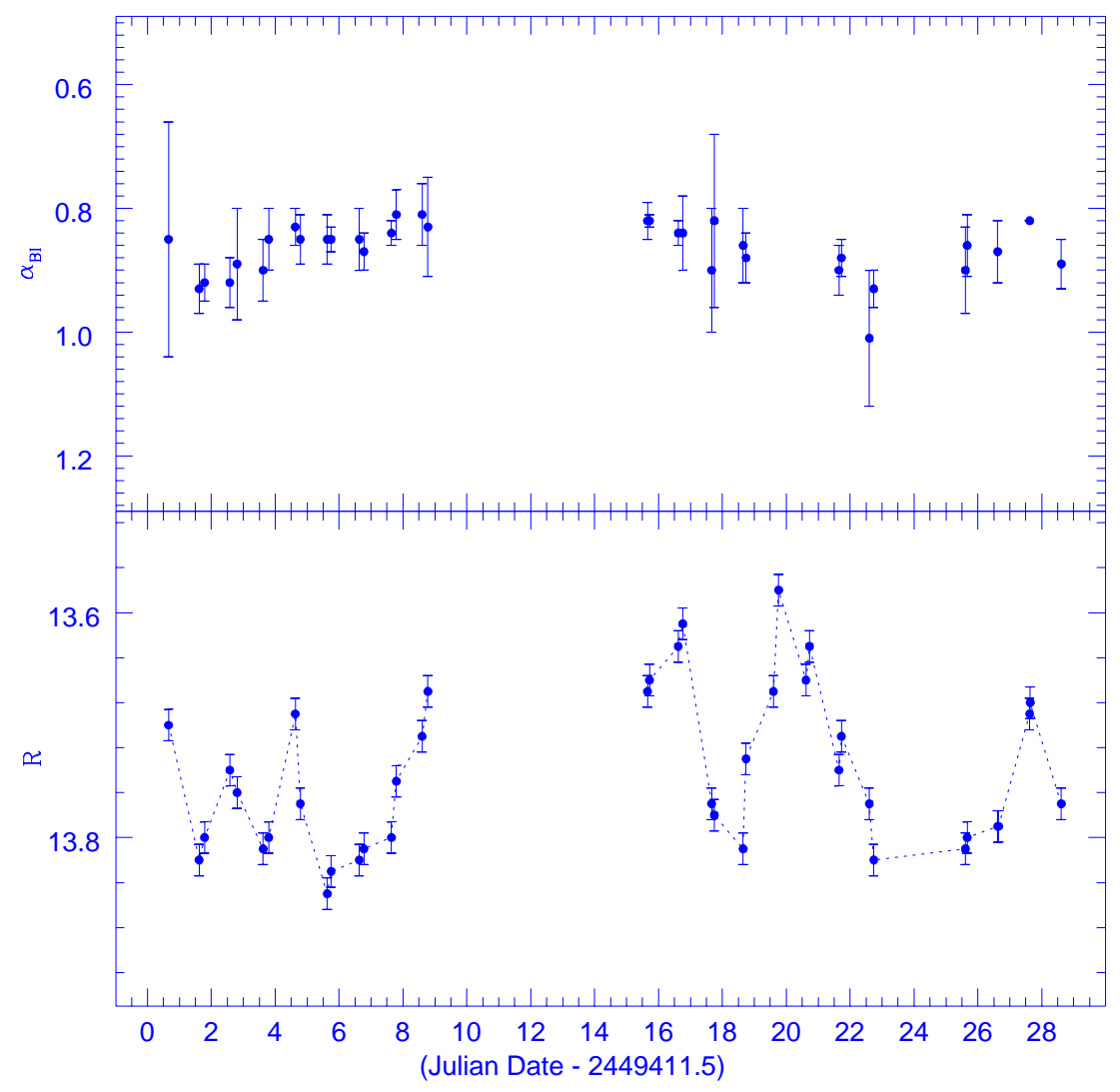

Fig. 4. The light curve in the $R$ passband (lower panel) is compared with the corresponding optical spectral index $\alpha_{B I}$ (upper panel)

run. However, it turned out that practically each one of these fluctuations is due to one of the comparison stars, since the simultaneous DLC relative to the other comparison star does not show the corresponding fluctuation. The only exception is a 0.15 mag jump seen at $\sim 16.5$ UT on March 3; however, its reality is uncertain since the feature is a single data point seen only on the $V$-band DLC (Fig. 1). Thus, the availability of differential light curves using multiple comparison stars serves as a powerful discriminator and a safeguard against serious misinterpretation of the data. It may be noted that the amplitudes of these ultra-rapid fluctuations are typically of order of a few percent and thus the present optical data of 1994 do not exclude the possibility of the blazar exhibiting lower level ultra-rapid variations, similar to those inferred by Wagner et al. (1996) from the statistical analysis of the 1990 light curves of this blazar.

\section{Conclusions}

The dense temporal $B, V, R$ and $I \mathrm{CCD}$ monitoring of the variable blazar S5 $0716+71$ is presented over a total duration of about 4 -weeks. These observations along with the optical monitoring carried out by Wagner et al. (1996)
Table 5. Rapid intensity fluctuation events seen on the DLCs, and the comparison stars most likely responsible for the events

\begin{tabular}{ccclc}
\hline $\begin{array}{c}\text { Date } \\
\text { March 94 }\end{array}$ & $\begin{array}{c}\text { UT } \\
\text { (hours) }\end{array}$ & $\begin{array}{c}\text { Amplitude } \\
\text { (mag) }\end{array}$ & Filter(s) & $\begin{array}{c}\text { Responsible } \\
\text { star }\end{array}$ \\
\hline & 18.8 & 0.06 & $I$ & 1 \\
15 & 17.1 & 0.05 & $V$ & 2 \\
16 & 17.2 & 0.03 & $B, V, R, I$ & 2 \\
17 & 18.5 & 0.05 & $R$ & 3 \\
19 & $>17$ & 0.10 & $R, I$ & 1 \\
21 & 16.3 & 0.03 & $R, I$ & 2 \\
22 & 15.2 & 0.05 & $B$ & 1 \\
22 & 16.9 & 0.06 & $V, R, I$ & 2 \\
25 & 15.8 & 0.05 & $B, V, R, I$ & 1 \\
26 & 15.5 & 0.06 & $R, I$ & 2 \\
28 & $\sim 15$ & 0.05 & $R$ & 2 \\
\hline
\end{tabular}

and by Ghisellini et al. (1997) have been used to derive the following useful results:

1. The present light curves in all four passbands show tight correlation. This indicates that the optical spectrum of the flaring component is similar to that of the base emission. It appears that the blazar outbursts are superposed on a constant base level emission. 


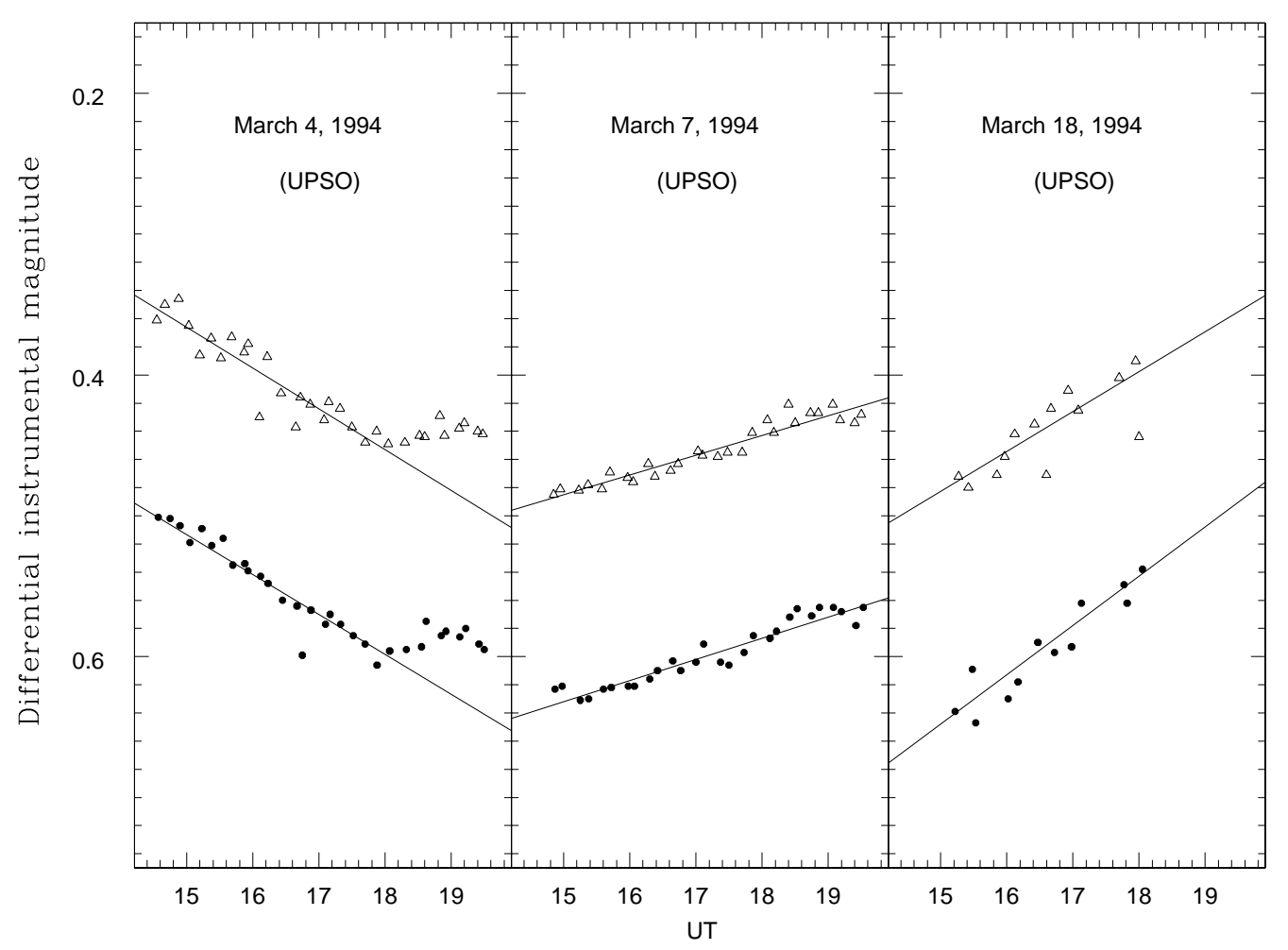

Fig. 5. DLCs relative to the comparison star 1 in the $R$ and $I$ passbands for those three nights when the blazar intensity varied by $\gtrsim 0.01 \mathrm{mag}$ per hour for a minimum of 2 hours. The data for the remaining 2 passbands have not been plotted as the temporal coverage was not so dense as in $R$ and $I$, due to lower sensitivity of the CCD. Note that the positive bump seen in the profiles of March 4 near 19 UT is clearly due to a variation of the comparison star (see Fig. 1) and therefore has been ignored in the computation of the best fit-line

2. The optical spectral index of the blazar varies between 0.81 to 1.01 with a mean value of 0.84 and has no dependence on the flux.

3. No clear event of ultra-rapid fluctuations of the blazar on time scale $\leq 1$ hour was detected during the present campaign.

4. Events with large amplitude ( $\geq 1 \mathrm{mag}$ ) brightening or fading have almost the same duration of about 30 days. Present observations have been carried out when the source was in a low optical state.

5. The prominent events of intra-night variability are well fitted with an exponential intensity profile with essentially the same rate of variations in all the optical passbands.

Acknowledgements. We gratefully acknowledge the useful discussions with P.J. Wiita. Thanks are also due to anonymous referee for useful comments.

\section{References}

Antonucci R.R.J., Hickson P., Olszewski E.W., Miller J.S., 1986, AJ 92, 1

Biermann P.L., Duerbeck H., Eckart A., et al., 1981, ApJ 247, L53
Ghisellini G., Villata M., Raiteri C.M., et al., 1997, A\&A 327, 61

Gopal-Krishna, Sagar R., Wiita P.J., 1993, MNRAS 262, 963

Gopal-Krishna, Sagar R., Wiita P.J., 1995, MNRAS 274, 701

Guilbert P.W., Fabian A.C., Rees M.J., 1983, MNRAS 205, 593

Howell S.B., 1989, PASP 101, 616

Mohan V., Paliwal D.C., Mahra H.S., 1991, BASI 19, 235

Qian S.J., Li X.C., Wegner R., Witzel A., Krichbaum T.P., 1996, Chin. Astron. Astrophys. 20, 15

Quirrenbach A., Witzel A., Wagner S., et al., 1991, ApJ 372, L71

Sagar R., Pati A.K., 1989, BASI 17, 6

Schalinski C.J., Witzel A., Krichbaum T.P., et al., 1992, in: Valtaoja E., Valtonen M. (eds.) Variability in Blazars. Cambridge Univ. Press, p. 221

Takalo L.O., Sillanpää A., Nilsson K., 1994, A\&AS 102, 497

Urry C.M., Maraschi L., Edelson R., et al., 1993, ApJ 411, 614

Villata M., Raiteri C.M., Lanteri L., Sobrito G., Cavallone M., 1998, A\&AS 130, 305

Wagner S.J., 1991, in "Variability of Active Galaxies", Springer Lecture Notes on Physics, Vol. 377, Duschl W., Wagner S. \& Camenzind M. (eds.), p. 163

Wagner S.J., Witzel A., Krichbaum T.P., et al., 1993, A\&A 271,344

Wagner S.J., Witzel A., Heidt J., et al., 1996, AJ 111, 2187 